\title{
ZERO-DIMENSIONALITY OF SOME PSEUDOCOMPACT GROUPS
}

\author{
DIKRAN DIKRANJAN
}

(Communicated by Franklin D. Tall)

\begin{abstract}
We prove that hereditarily disconnected countably compact groups are zero-dimensional. This gives a strongly positive answer to a question of Shakhmatov. We show that hereditary or total disconnectedness yields zerodimensionality in various classes of pseudocompact groups.
\end{abstract}

\section{INTRODUCTION}

Throughout ihis paper all topological groups are assumed to be Hausdorff. For a topological group $G$ the quasi-component $q(G)$ of the neutral element 1 of $G$ is the intersection of all clopen (= closed and open) sets containing 1 . We denote by $c(G)$ the connected component of 1 in $G$ and by $\tilde{G}$ the completion of $G$. The group $G$ is hereditarily disconnected if $c(G)=\{1\}$, totally disconnected if $q(G)=\{1\}$ (i.e., every two points can be separated by a partition), and zero-dimensional if the topology of $G$ has a base of clopen sets [E]. Zero-dimensionality yields total disconnectedness, and total disconnectedness yields hereditary disconnectedness for arbitrary topological spaces. The aim of this paper is to investigate the reverse implications

hereditarily disconnected $\stackrel{(\mathrm{i})}{\Longrightarrow}$ totaliy disconnected $\stackrel{(2)}{\Longrightarrow}$ zero-dimensional which in general fail to be true (see Sierpiński [S], Knaster and Kuratowski [KK], and Mazurkiewicz [M] for topological spaces). Erdős [Er] produced the first example of a totally disconnected topological group which is not zero-dimensional (see van Mill [vM] and Ursul [U2] for stronger results), and Ursul [U1] disproved (1) by means of an appropriate subgroup of $\mathbb{R}^{2}$. On the other hand, for locally compact groups hereditary disconnectedness implies zero-dimensionality (see Fact 2.2). This fact suggests that we restrict our investigation within appropriate classes of compact-like groups. We consider the following generalizations of compactness for a topological group $G$ : precompact (i.e., $\tilde{G}$ is compact), pseudocompact (every continuous function $G \rightarrow \mathbb{R}$ is bounded), hereditarily

Received by the editors July 29, 1992; presented to the Italian Group of Topology $40 \%$ at the meeting of May 28, 1992, in Vietri sul Mare, Salerno.

1991 Mathematics Subject Classification. Primary 54F45, 54D05; Secondary 22A05, 54D25, 54D30.

Key words and phrases. Connected group, (totally, hereditarily) disconnected group, zero-dimensional group, connected component, quasi-component, (hereditarily) pseudocompact group, countably compact group, (totally) minimal group, torsion group. 
pseudocompact (every closed subgroup of $G$ is pseudocompact), countably compact (eacti open countable cover of $G$ admits a finite subcover), minimal (each continuous isomorphism $G \rightarrow H$ is open), totally minimal (each Hausdorff quotient of $G$ is minimal). We remind the reader that each compact group is totally minimal and countably compact (the converse is also true in the abelian case [DSh1]), each countably compact group is hereditarily pseudocompact, and each pseudocompact group is precompact [CRs, Theorem 1.1]. The totally minimal groups are precisely the groups satisfying the open mapping theorem; i.e., each continuolis surjective homomorphisin $G \rightarrow H$ is open [DP]. Prodanov and Stoyanov proved that abelian minimal groups are precompact (for more information on minimal and totally minimal groups see [DPS], and for the impact of pseudocompactness on minimality see [DSh1] and [DSh2]). Mariy examples of hereditarily pseudocompact noncountably compact groups can be found in [D4], where hereditary pseudocompactness is characterized within the class of totally minimal abelian groups. For any precompact group $G$ zerodimensionality of $G$ is equivalent to $\operatorname{dim} G=0$, where $\operatorname{dim} G$ denotes the covering Čech-Lebesgue dimension of $G$ [Sh1, Corollary 3.4].

Comfort and van Mill [CvM, Corollary 7.7] disproved (2) for pseudocompact abelian groups and asked if (1) is true for precompact groups. The author answered negatively for the smaller class of pseudocompact abelian groups [D1, Corollary 3.7] and showed that under the assumption $2^{\omega_{1}}=2^{\omega}$ the counterexamples can be chosen even totally minimal. This set-theoretical assumption was removed in [D2, Theorem 7] where further evidence was given for the strong failure of (1) even in the class of totally minimal pseudocompact groups. On the other hand, Shakhmatov [Sh2] proved (2) for minimal pseudocompact groups (see Corollary 1.6 below) and asked if (1) holds for countably compact groups:

1.1. Question (Shakhmatov [CHR, Question 3.2A.4]). Is every hereditarily disconnected, countably compact group totally disconnected?

A positive answer to this question was announced in [D2, Theorem 6] (see Corollary 1.3). The main result of the present paper answers positively Question 1.1 by showing that both (1) and (2) hold in the larger class of hereditarily pseudocompact groups.

1.2. Theorem. Let $G$ be a hereditarily pseudocompact group. Then $q(G)=$ $c(G)$ and $G / c(G)$ is zero-dimensional. In particular, every hereditarily disconnected, hereditarily pseudocompact group is zero-dimensional.

In particular, both (1) and (2) are true in the smaller class of countably compact groups.

1.3. Corollary [D2, Corollary 5 a), Theorem 6]. Let $G$ be a countably compact group. Then $q(G)=c(G)$ and $G / c(G)$ is zero-dimensional. In particular, every countably compact hereditarily disconnected group is zero-dimensional.

This gives a positive answer to Question 1.1.

1.4. Corollary. Every hereditarily disconnected, countably compact group is totally disconnected.

The following description of the quasi-component will be crucial in our exposition. It provides also a new proof of Shakhmatov's result. 
1.5. Proposition [D1, Lemma 1.4; D3, Theorem 2.2]. For every topological group $G$ the quasi-component $q(G)$ is a closed normal subgroup of $G$ and the group $G / q(G)$ is totally disconnected. If $G$ is pseudocompact, then $q(G)$ coincides with the intersection of all open normal subgroups of $G$. More precisely, $q(G)=G \cap c(\tilde{G})$.

1.6. Corollary [Sh2]. Let $G$ be a pseudocompact totally disconnected group. Then $G$ admits a coarser zero-dimensional group topology. In particular, a minimal pseudocompact Abelian group $G$ is totally disconnected iff $G$ is zerodimensional.

Proof. By the above proposition the intersection of all open normal subgroups of $G$ is trivial; thus, the group topology $\sigma$ on $G$ having as a base of neighborhoods of 1 the family of all open normal subgroups of $G$ is a Hausdorff group topology on $G$ coarser than the one given. If $G$ is minimal, then $\sigma$ coincides with the topology of $G$. It remains to note that $\sigma$ is zero-dimensional since every open subgroup is closed.

As already noted, here total disconnectedness cannot be replaced by hereditary disconnectedness even if the group $G$ is totally minimal.

Another proof of Corollary 1.6 is provided by setting $q(G)=1$ in the following theorem announced in [D2, Theorem 3].

1.7. Theorem. Let $G$ be a pseudocompact group. Then $G / q(G)$ admits a coarser zero-dimensional group topology. Moreover, for the conditions:

(a) $G / q(G)$ is zero-dimensional;

(b) $q(G)$ is dense in $c(\widehat{G})$;

(c) $G / q(G)$ is minimal;

the implications (c) $\Rightarrow(\mathrm{b}) \Longleftrightarrow$ (a) hold. If $G$ is minimal and abelian, then they are all equivalent.

According to Theorem 1.7 (or Corollary 1.6) $G / q(G)$ is zero-dimensional for a totally minimal pseudocompact group $G$. This should be compared with Theorem 1.2, where a stronger conclusion is obtained with total minimality replaced by hereditary pseudocompactness. It is not difficult to see that for a topolgical group $G c(G)=q(G)$ whenever $G / c(G)$ is zero-dimensional. Let $\mathfrak{P}$ be a class of topological groups closed under taking quotient groups. Then the implication (1) holds in $\mathfrak{P}$ iff the quasi-component and the connected component coincide in each group of $\mathfrak{P}$. More details concerning the relation between the quasi-component and the connected component of a topological group can be found in [D1-D3].

The next result is of a different nature-the algebraic structure of the underlying abstract group may imply zero-dimensionality for a pseudocompact group. Recall that a group $G$ is torsion if for every $g \in G$ there exists a power with $g^{n}=1$ and $n \neq 0$.

\subsection{Theorem. Every torsion pseudocompact group is zero-dimensional.}

The proofs of Theorems 1.2, 1.7, and 1.8 are given in $\S 2$. Those of 1.2 and 1.8 are based on Proposition 1.5, on an easy zero-dimensionality criterion (2.3), on Theorem 1.7, and on an appropriate "approximation" technique for pseudocompact groups (see 2.4-2.6). In the abelian case Theorem 1.8 follows 
from [CR, (7.4)] (see also [DSh3, Theorem 2.9] where the result is announced explicitly). A stronger version of 1.8 can be obtained involving a stronger form of Lemma 2.3, but this will require more algebra (namely, in both 2.3 and 1.8 "torsion" can be replaced by a weaker condition without affecting the proofs; more details can be found in the comments following Lemma 2.3).

Our method does not permit us to treat strongly pseudocompact groups (i.e., pseudocompact groups containing dense countably compact subset). However, Theorem 1.2 permits us to hope that zero-dimensionality of hereditarily disconnected strongly pseudocompact groups can also be expected, so we have, in the spirit of Question 1.1, the following

1.9. Question. Is every strongly pseudocompact, hereditarily disconnected group zero-dimensional?

This was the version of Theorem 1.2 with hereditary pseudocompactness replaced by strong pseudocompactness. One can consider also the following weaker form.

1.10. Question. Is every strongly pseudocompact, totally disconnected group zero-dimensional?

Clearly, "yes" to 1.9 yields "yes" to 1.10 and $c(G)=q(G)$ for every strongly pseudocompact group $G$.

It is known that quotients of zero-dimensional pseudocompact groups are zero-dimensional [T, Corollary 3]. The counterpart concerning the total disconnectedness may fail in a strong way. In fact, every connected pseudocompact group $K$ is a quotient of a totally disconnected pseudocompact group $K_{t}$ of the same dimension with $w\left(K_{t}\right)=\max \left\{w(K), \omega_{1}\right\}$ [D2, Theorem 15; D3, Corollary 4.3]. On the other hand, Theorem 1.2 yields trivially that quotients of hereditarily disconnected, hereditarily pseudocompact group are totally disconnected, even zero-dimensional. This justifies the following

1.11. Question. (a) Is the quotient of a hereditarily disconnected strongly pseudocompact group hereditarily disconnected ?

(b) Is the quotient of a totally disconnected strongly pseudocompact group totally disconnected?

(c) Is the quotient of a totally disconnected strongly pseudocompact group hereditarily disconnected?

Obviously, "yes" to 1.9 yields "yes" to all (a)-(c), while "yes" to 1.10 yields "yes" to (b) and (c).

\section{Proofs of THE MAIN Results}

The notation and terminology follow [HR, DPS, E] (the term "hereditarily disconnected" adopted in [E] seems to be not universally accepted, our choice was determined by the lack of a reasonably compact term for the property “ $q(G)=1 ")$. All groups are multiplicative, in particular, the neutral element of a group is always denoted by 1 . If $X$ is a subset of a topological group $G$, then $\langle X\rangle$ is the smallest subgroup of $G$ that contains $X$ and $\bar{X}$ is the closure of $X$. We denote by $\mathbb{Z}$ the integers and by $\mathbb{R}$ the reals.

The following notion is needed to characterize the pseudocompact groups: a subset $Y$ of topological space $X$ is $G_{\delta}$-dense if $Y$ meets every nonempty $G_{\delta}$-set of $X$. 
2.1. Fact [CRs, Theorems 1.2 and 4.1]. Let $G$ be a precompact group. Then the following are equivalent: (1) $G$ is pseudocompact; (2) $G$ is $G_{\delta}$-dense in $\tilde{G}$; and (3) $\tilde{G}=\beta G$.

2.2. Fact [HR, (7.7), (7.8)]. For every locally compact group $G, c(G)=q(G)$ and $G / c(G)$ is zero-dimensional.

Concerning the next zero-dimensionality criterion we observe that for a compact group $G$ and a closed normal subgroup $L$ of $G$, the quotient $G / L$ is metrizable iff the subgroup $L$ is $G_{\delta}$.

2.3. Lemma. Let $G$ be a compact group with connected component $C$. If for every closed normal $G_{\delta}$-subgroup $L$ of $C$ the quotient $C / L$ is torsion, then $G$ is zero-dimensional.

Proof. According to Fact 2.2 it suffices to show that $C=1$. Assume that $C \neq 1$. To get a contradiction, it is enough to provide a metrizable nontorsion quotient of $C$. By Peter-Weyl's theorem there exist $n$ and a nontrivial continuous homomorphism $f: C \rightarrow U(n)$ into the group of $n \times n$ unitary matrices [HR, (22.14)]. Since $C$ is connected, the subgroup $f(C)$ of $U(n)$ is a connected compact Lie group which is clearly metrizable as a subgroup of $U(n)$. Moreover, the compactness of $C$ yields that $f(C)$ is a quotient of $G$. Since every connected compact Lie group contains a copy of $\mathbb{R} / \mathbb{Z}$, the group $f(C)$ is nontorsion.

The reader should have noted that the assumption that $G$ is not zero-dimensional in the above proof produces a quotient $f(C)$ of $C$ with a stronger property than just being nontorsion. It can be shown that the group $f(C)$ contains either a copy of the free group $F_{\mathfrak{c}}$ of rank $\mathfrak{c}=2^{\omega}$ or a copy of the free abelian group $\mathbb{Z}^{(\mathfrak{c})}$ of rank $\mathfrak{c}$ [D3, Theorem 3.7]. Hence, in the above lemma, as well as in Theorem 1.8, "torsion" can be replaced by the weaker condition "the group contains copies of neither $F_{\mathfrak{c}}$ nor $\mathbb{Z}^{(\mathfrak{c})}$ " (i.e., the group has free-rank less than $\mathfrak{c}$ ).

Proof of Theorem 1.7. The first assertion follows from Proposition 1.5 arguing as in the proof of Corollary 1.6 and taking the quotient topology on $G / q(G)$ with respect to $\sigma$. It can be obtained also directly from Corollary 1.6.

(a) $\Rightarrow$ (b) It was proved by Tkačenko [T, Corollary 1] that all three dimensions coincide for a pseudocompact group $G$ :

$$
\operatorname{dim} G=\operatorname{ind} G=\operatorname{Ind} \beta G .
$$

Moreover, he proved that for every closed normal subgroup $N$ of $G$

$$
\operatorname{dim} G=\operatorname{dim} \bar{N}^{\tilde{G}}+\operatorname{dim} G / N
$$

holds. Let $Q=\overline{q(G)}^{\tilde{G}}$. By (1) applied to the closed subgroup $q(G)$ of $G$ and our hypothesis we have

$$
\operatorname{dim} G=\operatorname{dim} Q+\operatorname{dim} G / q(G)=\operatorname{dim} Q .
$$

According to Fact $2.1 \tilde{G}=\beta G$; hence, $\operatorname{dim} G=\operatorname{dim} \tilde{G}$ [E, 7.1.17]. Now (2) gives $\operatorname{dim} \tilde{G}=\operatorname{dim} Q$. On the other hand, (1) applied to the group $\tilde{G}$ and its closed subgroup $Q$ gives $\operatorname{dim} \tilde{G}=\operatorname{dim} Q+\operatorname{dim} \tilde{G} / Q$; thus, $\tilde{G} / Q$ is zerodimensional. In particular, $\tilde{G} / Q$ is hereditarily disconnected. Consider the 
canonical homomorphism $\varphi: \tilde{G} \rightarrow \tilde{G} / Q$. The image of the connected subgroup $c(\tilde{G})$ under $\varphi$ should be connected and, hence, trivial by the hereditary disconnectedness of $\tilde{G} / Q$. This proves the inclusion $c(\tilde{G}) \subseteq Q$. The inverse inclusion follows from the inclusion $q(G) \subseteq c(\tilde{G})$ due to Proposition 1.5.

(b) $\Rightarrow$ (a) Following the above notation we note that according to SulleyGrant's lemma [DPS, Lemma 4.3.2] the restriction $\psi=\varphi \uparrow_{G}: G \rightarrow \varphi(G)$ is open iff $c(\tilde{G})=\overline{c(\tilde{G}) \cap G}$, i.e., (b) holds. By Proposition 1.5 this is equivalent to $c(\tilde{G})=Q$. This is equivalent also to the fact that the obvious continuous algebraic isomorphism $i: G / q(G) \rightarrow \varphi(G)$ is open when $G / q(G)$ is equipped with the quotient topology and $\varphi(G)$ is equipped with the topology induced by $\tilde{G} / Q$. In particular, (b) yields that the completion of $G / q(G)$ is isomorphic to $\tilde{G} / c(\tilde{G})$ and so is zero-dimensional (Fact 2.2). Hence $G / q(G)$ is zero-dimensional as a subspace of a zero-dimensional space [E, 6.2.11].

(c) $\Rightarrow$ (b) Assume that $G / q(G)$ is minimal. Then the isomorphism $i$ defined in the above paragraph is obviously open, so that the morphism $\psi$ is open as a composition of $i$ and the open canonical homomorphism $G \rightarrow G / q(G)$. As noted above, this yields (b). This implication can be obtained also directly from Corollary 1.6 and the implication $(a) \Rightarrow(b)$.

Now assume that $G$ is minimal and abelian. Then according to [DPS, Exercise 4.5.15] (c) $\Longleftrightarrow$ (b).

\subsection{Lemma. Let $N$ be a compact totally disconnected group, $C$ a compact} connected metrizable group, and $G$ a dense hereditarily pseudocompact subgroup of $N \times C$. Then $G$ contains the subgroup $\{1\} \times C$.

Proof. Consider first the case when $C$ is abelian. Then $C$ is monothetic, i.e., there exists $y \in C$ such that the cyclic subgroup generated by $y$ is dense in $C$ (see [HR, (25.14)]). Let $p: N \times C \rightarrow C$ be the canonical projection. Then $p(G) \subseteq C$ is a dense pseudocompact subgroup of the metrizable group $C$; hence, $p(G)$ is compact [E]. Therefore, $p(G)$ is also closed in $C$, and consequently $p(G)=C$. Then there exists $x \in N$ such that $(x, y) \in G$. Now $\overline{\langle x\rangle}$ is a closed subgroup of $N$ and, thus, compact, totally disconnected, and monothetic. Thus $\overline{\langle x\rangle}$ is metrizable (see [HR, (25.16)]). Hence $\overline{\langle x\rangle} \times \overline{\langle y\rangle}$ is metrizable as well. Then also $B=\overline{\langle(x, y)\rangle}$ is metrizable, so that $B \cap G$ is metrizable. But $B \cap G$ is a closed subgroup of $G$; hence, it is also pseudocompact; thus, $B \cap G$ is compact. Since $\langle(x, y)\rangle \subseteq B \cap G$, this gives $B=\overline{\langle(x, y)\rangle} \subseteq B \cap G$; hence, $B \subseteq G$. Now it is enough to show that $\{1\} \times C \subseteq B$.

By $y=p(x, y) \in p(B)$, by the density of $\langle y\rangle$ in $C$, and by the continuity of $p$ it follows that $C=\overline{p(B)}$. Now the compactness of $B$ and the continuity of $p$ yield that $p(B)$ is compact as well; thus, $p(B)$ is closed and consequently $C=p(B)$. Let $p^{\prime}: B \rightarrow C$ be the restriction of $p$ on $B$. We show that

$$
C=p^{\prime}(c(B)) \text {. }
$$

Clearly $p^{\prime}$ is surjective, so that $p^{\prime}$ induces a surjective continuous homomorphism $p^{\prime \prime}: B / c(B) \rightarrow C / p^{\prime}(c(B))$ of compact groups. These quotients make sense since the groups $B$ and $C$ are monothetic and, hence, abelian. By Fact 2.2 the group $B / c(B)$ is zero-dimensional. Since quotients of zero-dimensional compact groups are zero-dimensional (see [T, Corollary 3] for pseudocompact groups), it follows that the image $C / p^{\prime}(c(B))$ of $p^{\prime \prime}$ is zero-dimensional. On 
the other hand, it obviously is connected, as a quotient of the connected group $C$. This is possible only if the group $C / p^{\prime}(C(B))$ is trivial. Thus (3) holds. To check the inclusion $\{1\} \times C \subseteq B$ take $c \in C$; then by (3) there exists $b \in c(B)$ such that

$$
c=p^{\prime}(b) \text {. }
$$

Since $c(B) \subset B \cap(\{1\} \times C)$, it follows that $b=(1, v)$ for some $v \in C$. Now (4) gives $v=c$; thus, $(1, c)=b \in B$.

Consider now the general case. Then $C$ is covered by compact connected abelian subgroups (in case $C$ is a Lie group take the maximal tori in $C$, for the general case see, for example, [My]). Let $x$ be an element of $C$, and let $C_{1}$ be a compact connected abelian subgroup of $C$ containing $x$. Since $C$ is metrizable, $C_{1} \subseteq C$ is a $G_{\delta}$-set. Thus $N \times C_{1} \subseteq N \times C$ is a closed $G_{\delta}$-set. By Fact 2.1 $G_{1}=G \cap\left(N \times C_{1}\right)$ is a dense subgroup of $N \times C_{1}$. Moreover, $G_{1}$ is hereditarly pseudocompact as a closed subgroup of $G$. It follows from the above proof in the abelian case that $1 \times C_{1} \subseteq G_{1}$, so we get $(1, x) \in G_{1} \subseteq G$. This proves the inclusion $1 \times C \subseteq G$.

In the next lemma we show that general pseudocompact groups can be approximated in a certain sense by pseudocompact groups with additional nice properties.

2.5. Lemma. Let $G$ be a pseudocompact group, and let $C$ be the connected component of $\tilde{G}$. Then for every closed normal $G_{\delta}$-subgroup $L$ of $C$ there exists a closed normal $G_{\delta}$-subgroup $N$ of $\tilde{G}$, such that the subgroup $G_{1}=N C \cap G$ of $G$ satisfies the following conditions:

(a) $G_{1}$ is a closed normal $G_{\delta}$-subgroup of $G$ and $\tilde{G}_{1}=N C$;

(b) $L$ is a normal subgroup of $\tilde{G}_{1}$ and the following isomorphism of topological groups holds:

$$
\tilde{G}_{1} / L \cong N L / L \times C / L
$$

(c) there exists a surjective continuous homomorphism of $G_{1}$ onto $C / L$.

Proof. (a) There exists a $G_{\delta}$-subset $W$ of $\tilde{G}$ such that $W \cap C=L$. Let $N$ be a closed normal $G_{\delta}$-subgroup of $\tilde{G}$ such that $N \subset W$ [CR, Lemma 1.6(b)]. Then

$$
N \cap C \subset L .
$$

Clearly $N C=C N$ is a closed normal $G_{\delta}$-subgroup of $\tilde{G}$. Then in view of Fact $2.1 G_{1}=N C \cap G$ is a dense subgroup of $N C$; hence, $\tilde{G}_{1}=N C$. Obviously, $G_{1}$ is a closed normal $G_{\delta}$-subgroup of $G$.

(b) To show that $L$ is a normal subgroup of $\tilde{G}_{1}$ we see first that the subgroup $[N, C]$ of $\tilde{G}$ generateci by all commutators $[n, l]=n^{-1} l^{-1} n l$, where $n \in N$ and $l \in C$, satisfies

$$
[N, C] \subset L \text {. }
$$

In fact, let $n \in N$ and $l \in C$. Then $[n, l] \in N \cap C \subset L$ by (6) since both $N$ and $C$ are normal subgroups of $\tilde{G}$, so that $n^{-1} l^{-1} n \in C$ and $l^{-1} n l \in N$. In particular, (7) yields $[N, L] \subset L$, so that $L$ is a normal subgroup of $\tilde{G}_{1}=N C$. 
Denote by $\varphi: N C \rightarrow N C / L$ the canonical homomorphism. To establish the isomorphism (5) we identify in the obvious way the quotient groups $N L / L$ and $C / L$ with the subgroups $\varphi(N L)$ and $\varphi(C)$ respectively of $N C / L$ equipped with the induced topology. Hence we have to see that $N C / L$ is a direct product of its subgroups $\varphi(N L)$ and $\varphi(C)$. To define the algebraic isomorphism (5), we note that after application of $\varphi$ to the identities $\tilde{G}_{1}=(N L) C$ and $N L \cap C=L$ (the second one follows from (6)) we get $\varphi(N L) \varphi(C)=N C / L$ and $\varphi(N L) \cap$ $\varphi(C)=1$. Moreover, (7) yields $[N L, C] \subseteq L$, so that the subgroups $\varphi(N L)$ and $\varphi(C)$ commute eiementwise. This establishes the algebraic isomorphism

$$
\mu: \varphi(N L) \times \varphi(C) \longrightarrow \varphi(N L) \varphi(C)=N C / L
$$

defined by $\mu(x, y)=x y$. Now $\mu^{-1}$ modulo the above identifications will give (5). Let us verify that the isomorphism $\mu$ is also topological whenever the product topology is taken on the left-hand side of (8). It is important to note that all groups in question are compact, so that it suffices to see, for example, that $\mu$ is continuous. To this end take a neighborhood $W_{0}$ of 1 in $N C / L$ and choose another neighborhood $W$ of 1 in $N C / L$ such that $W^{2} \subseteq W_{0}$. Then $U=W \cap \varphi(N L)$ and $V=W \cap \varphi(C)$ are neighborhoods of 1 in $\varphi(N L)$ and $\varphi(C)$, such that $\mu(U \times V) \subseteq W^{2} \subseteq W_{0}$.

(c) Consider the continuous homomorphism $p: G_{1} \rightarrow C / L$ obtained as the restriction on $G_{1}$ of the composition of $\varphi$ and the projection $N C / L=$ $\tilde{G}_{1} / L \rightarrow C / L$ in (5). Being pseudocompact and dense in the metrizable group $C / L$, the subgroup $p\left(G_{1}\right)$ must coincide with $C / L$. Thus $p$ is surjective.

The following lemma prepares the final step for the proof of Theorems 1.2 and 1.8 .

2.6. Lemma. Let $G$ be a hereditarily pseudocompact group and $C$ be the connected component of $\tilde{G}$. Then $C \subseteq q(G) L$ holds for every closed normal $G_{\delta^{-}}$ subgroup $L$ of $C$.

Proof. Let $L$ be a closed normal $G_{\delta}$-subgroup of $C$. There exists a closed normal $G_{\delta}$-subgroup $N$ of $\tilde{G}$ such that the subgroup $G_{1}=N C \cap G$ of $G$ satisfies conditions (a)-(c) from Lemma 2.5. Moreover, $G_{1}$ is hereditarly pseudocompact as a closed subgroup of $G$. Denote by $\varphi: \tilde{G}_{1} \rightarrow \tilde{G}_{1} / L$ the canonical homomorphism. Then $G_{2}=\varphi\left(G_{1}\right)$ is a dense hereditarily pseudocompact subgroup of $\tilde{G}_{1} / L \cong N L / L \times C / L$. Obviously $C / L$ is connected and metrizable. On the other hand, the isomorphism (5) yields $N L / L \cong\left(\tilde{G}_{1} / L\right) /(C / L) \cong \tilde{G}_{1} / C$. The latter group is isomorphic to a subgroup of the totally disconnected group $\tilde{G} / C$ (Fact 2.2), so $N L / L$ is totally disconnected. Hence by Lemma $2.4 G_{2}$ contains $1 \times C / L$. Thus $C \subseteq G_{1} L$. By the modular law $\left(G_{1} \cap C\right) L \supseteq G_{1} L \cap C=C$ (in fact, choose an arbitrary $c \in G_{1} L \cap C$; then $c=g_{c} l_{c}$ for some $g_{c} \in G_{1}$ and $l_{c} \in L$; now $L \subseteq C$ yields $g_{c} \in C$ and, hence, $\left.g_{c}=C \cap G_{1}\right)$. It remains now to recall that according to Proposition $1.5 q(G)=G \cap C \supseteq G_{1} \cap C$.

Proof of Theorem 1.2. Let $G$ be a hereditarily pseudocompact group. We establish first the equality $q(G)=c(G)$. To this end it suffices to see that $q(G)$ is connected. Being a closed subgroup of $G, q(G)$ is pseudocompact. According to Lemma $2.6 q(G)$ is dense in $c(\tilde{G})$ since every neighborhood of 1 in $c(\tilde{G})$ contains a closed normal $G_{\delta}$-subgroup $L$ of $c(\tilde{G})$ [CR, Lemma 1.6(b)]. By 
Fact $2.1 c(\tilde{G})=\beta q(G)$; hence, the connectedness of $q(G)$ follows from the connectedness of $c(\tilde{G})$ [E, Theorem 6.1.14].

Finally, the density of $q(G)$ in $c(\tilde{G})$ yields, according to Theorem 1.7 and the equality $q(G)=c(G)$, that $G / c(G)$ is zero-dimensional.

Proof of Theorem 1.8. Let $G$ be a hereditarily pseudocompact group. Denote by $C$ the connected component of $\tilde{G}$. Let us see that for each closed normal $G_{\delta}$-subgroup $L$ of $C$ the quotient $C / L$ is torsion. By Lemma 2.5 there exists a subgroup $G_{1}$ of $G$ and a continuous surjective homomorphism of $G_{1}$ onto the group $C / L$. Now we observe that the group $G_{1}$ is torsion as a subgroup of the torsion group $G$. Hence the quotient group $C / L$ is torsion as well. Now, according to Lemma $2.3 \tilde{G}$ is zero-dimensional. Therefore, $G$ is zerodimensional as a subspace of a zero-dimensional space [E, 6.2.11].

\section{ACKNNOWLEDGMENTS}

The author is thankful to W. W. Comfort, K. H. Hofmann, and D. Remus for providing a preliminary version of [CHR] and to D. B. Shakhmatov for providing the original proof of Corollary 1.6 in October 1990. The author's thanks go also to the referee for her/his helpful suggestions concerning the original version of the paper.

\section{REFERENCES}

[CHR] W. W. Comfort, K. H. Hofmann, and D. Remus, A survey on topoligical groups and semigroups, Recent Progress in General Topology (M. Hušek and J. van Mill, eds.), NorthHolland, Amsterdam, 1992, pp. 58-144.

[CvM] W. W. Comfort and J. van Mill, Concerning connected, pseudocompact abelian groups, Topology Appl. 33 (1989), 21-45.

[CR] W. W. Comfort and L. C. Robertson, Extremal phenomena in certain classes of totally bounded groups, Dissertationes Math. (Rozprawy Mat.) 272 (1988).

[CRs] W. W. Comfort and K. A. Ross, Pseudocompactness and uniform continuity in topological groups, Pacific J. Math. 16 (1966), 483-496.

[D1] D. Dikranjan, Connectedness and disconnectedness in pseudocompact groups, Rend. Accad. Naz. Sci. XL Mem. Mat. 110 (1992), 211-221.

[D2] - Dimension et connexité des groupes pseudo-compacts, C. R. Acad. Sci. Paris Sér. I Math. 316 (1993), 309-314.

[D3] _ Quasi-component, dimension and connectedness in pseudocompact groups, preprint.

[D4] - Hereditarily pseudocompact groups (in preparation).

[DP] D. Dikranjan and Iv. Prodanov, Totally minimal topological groups. Annuaire Univ. Sofia Fac. Math. Méc. 69 (1974/75), 5-11.

[DPS] D. Dikranjan, Iv. Prodanov, and L. Stoyanov, Topological groups: characters, dualities and minimal group topologies, Monographs and Textbooks in Pure and Applied Mathematics (E. Taft and Z. Nashed, eds.), vol. 130, Marcel Dekker, New York and Basel, 1990.

[DSh1] D. Dikranjan and D. Shakhmatov, Compact-like totally dense subgroups of compact groups, Proc. Amer. Math. Soc. 114 (1992), 1119-1129.

[DSh2] __ Pseudocompact and countably compact Abelian groups: Cartesian products and minimality, Trans. Amer. Math. Soc. 335 (1993), 775-790.

[DSh3] __ Algebraic structure of the pseudocompact groups, Report 91-19, York University, Canada, 1991, pp. 1-37. 
[E] R. Engelking, General topology, Revised and completed edition, Sigma Ser. Pure Math., vol. 6, Heldermann, Berlin, 1989.

[Er] P. Erdős, The dimension of points in rational Hilbert space, Ann. of Math. (2) 41 (1940), 734-736.

[HR] E. Hewitt and K. Ross, Abstract hamonic analysis. I, Springer-Verlag, Berlin, Heidelberg, and New York, 1963.

[KK] B. Knaster and K. Kuratowski, Sur les ensembles connexes, Fund. Math. 2 (1921), 206-255.

[M] S. Mazurkiewisz, Sur les problémes $\kappa$ et $\lambda$ de Urysohn, Fund. Math. 10 (1927), 311-319.

[My] J. Mycielski, Some properties of connected compact groups, Colloq. Math. 5 (1958), $162-$ 166.

[vM] Jan van Mill, Totally disconnected n-dimensional group, Math. Japonica 32 (1986), 267273.

[Sh1] D. Shakhmatov, Imbeddings into topological groups preserving dimensions, Topology Appl. 36 (1990), 181-204.

[Sh2] _ Unpublished notes, October 1990.

[S] - Sur les ensembles connexes et non connexes, Fund. Math. 2 (1921), 81-95.

[T] M. Tkačenko, Dimension of locally pseudocompact groups and their quotient spaces, Comment. Math. Univ. Carolinae 31 (1990), 159-166.

[U1] M. Ursul, An example of a plane group whose quasi-component does not coincide with its component, Mat. Zametki 38 (1985), 517-522. (Russian)

[U2] _ On the dimension theory of topological fields, Izv. Akad. Nauk Moldav. SSR Ser. Fiz.-Tekhn. Mat. Nauk 3 (1987), 47-48. (Russian)

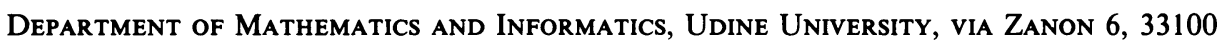
UDINE, ITALY

E-mail address: dikranja@udmi5400.cineca.iT 